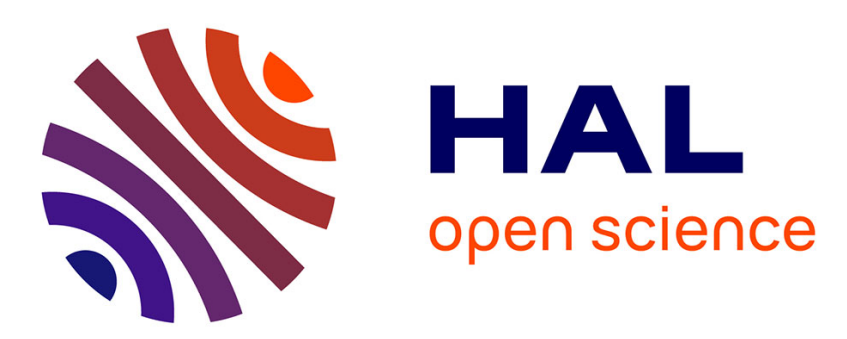

\title{
Broadcasting Based on Dominated Connecting Sets with MPR in a Realistic Environment for WSNs \& AD-HOC
}

\author{
Tarek Moulahi, Salem Nasri, Hervé Guyennet
}

\section{To cite this version:}

Tarek Moulahi, Salem Nasri, Hervé Guyennet. Broadcasting Based on Dominated Connecting Sets with MPR in a Realistic Environment for WSNs \& AD-HOC. Journal of Network and Computer Applications (JNCA), 2012, 35, pp.1720 - 1727. hal-00940257

\section{HAL Id: hal-00940257 \\ https://hal.science/hal-00940257}

Submitted on 31 Jan 2014

HAL is a multi-disciplinary open access archive for the deposit and dissemination of scientific research documents, whether they are published or not. The documents may come from teaching and research institutions in France or abroad, or from public or private research centers.
L'archive ouverte pluridisciplinaire HAL, est destinée au dépôt et à la diffusion de documents scientifiques de niveau recherche, publiés ou non, émanant des établissements d'enseignement et de recherche français ou étrangers, des laboratoires publics ou privés. 


\title{
Broadcasting Based on Dominated Connecting Sets with MPR in a Realistic Environment for WSNs \& AD-HOC
}

\author{
Tarek Moulahi ${ }^{\mathrm{a}, \mathrm{b}}$, Salem Nasri ${ }^{\mathrm{a}, \mathrm{c}}$, Hervé Guyennet ${ }^{\mathrm{b}}$, \\ ${ }^{a}$ CES ENIS Route Soukra km 4 B.P.:1173, 3000 Sfax, Tunisia. \\ ${ }^{b}$ LIFC UFR ST 16 route de Gray 25030 Besançon Cedex, France. \\ ${ }^{c}$ College of Computer, Q.U., P.B.: 6688, 51452 Buraydah, KSA.
}

\begin{abstract}
Broadcasting in wireless sensor networks (WSNs) is the dissemination of packets from one node to all nodes in the network. This flooding causes redundant data and broadcast storm problems. To counteract this effect, many methods have been introduced to minimize the redundancy, such as MPR (MultiPoint Relay) or DS-MPR (Connected Dominating Sets with MPR). These methods stipulate that a packet is correctly received if the receiver node is in the transmission radius of the sender node. But this fact is not always true, due to many factors like signal attenuation, noise and existence of obstacles. This paper focuses on DS-MPR. Firstly, we test it in a realistic environment to show its limit in terms of reachability. Secondly, we introduce a modification of DS-MPR to be applicable with a realistic physical layer. Our heuristic method is called RDS-MPR (Realistic DS-MPR). This heuristic has improved DS-MPR in reachability, which may exceed more than 94\%. Finally, we introduce an extension of RDS-MPR, called eRDS-MPR (extended RDS-MPR) which provides a reachability up to more than $97 \%$.
\end{abstract}

Keywords: Wireless Sensor Networks; Broadcasting; Connected Dominating Sets; MultiPoint Relay; Lognormal model.

\section{Introduction}

Recent advances in wireless communication technologies and the fabrication of low-cost wireless devices have led to the appearance of wireless sensor networks (WSNs). Due to the multi-functionality of sensors, WSNs have been utilized for a variety of applications such as environment monitoring, 
surveillance, and military. Moreover, WSNs can provide a good observation of nature and a high capacity of collecting information. However, the most important disadvantage of WSNs is their limit in energy. As a result, many methods, algorithms and protocols were introduced and developed to take into consideration this constraint. Since WSNs are considered ad-hoc networks with other characteristics, many algorithms and methods of ad-hoc networks $[24,25]$ could be reused and reconfigured according to the WSNs' specificity. In this paper, we focus our interest on broadcasting algorithms in WSNs and ad-hoc. Firstly, broadcasting means the packet distribution from a source sensor to all other destination sensors in the network. Many research projects have been developed discussing solutions to broadcast information in these kinds of networks and taking into account the minimization of energy consumption. Since the communication process consumes more energy compared to the others processes (i.e., sensing, communicating and processing), many methods and algorithms have focused on reducing the number of relay nodes in order to minimize the number of communication packets. This also minimizes the consumed energy and broadcast storm problems.

By studying currently broadcasting method, we find that the most of them are conceived in an ideal environment (i.e., they stipulate that a message send from a node is certainly received by another node if the receiver is in the sender covering area). This fact has negative consequences on communication and clearly decreases the accessibility of the broadcasting method used, because in a real environment there are signal attenuation, obstacles and nature factors. In this manuscript, an overview of DS-MPR is given. Thus, a modification of DS-MPR to be applicable with a realistic environment is proposed, aiming to increase the accessibility.

This paper is organized as follows: Section 2 outlines the most well-known methods of broadcasting and provides the problem definition of our topic. In Section 3, we perform a study of DS-MPR, and we show that this method is not a valid method of broadcasting because its reachability is less than $80 \%$ in the best cases. Section 4 presents RDS-MPR, which is an improvement of DS-MPR to be applicable with a realistic physical layer using the lognormal shadowing model (LNM). In LNM, a node correctly receives a packet from another node with a probability $p$. This probability is defined as a function of transmission radius, power attenuation factor and distance between nodes. In Section 5, an extension of RDS-MPR is proposed. This new method, called eRDS-MPR, greatly improves DS-MPR in reachability. Finally, in Section 6 , a performance study of the proposed methods compared to DS-MPR is 
given.

\section{Preliminaries}

\subsection{Related work}

To perform the task of broadcasting, pure flooding is used first. This method increases the number of redundant packets, broadcast storm problems and energy consumption. Subsequently, many research projects have focused on minimizing these problems. In literature, there is several methods and protocols for broadcasting in WSNs. Most of them are conceived in an ideal environment $[6,7,8,9,10]$, also called unit disk model (UDM). In UDM, a receiver node can correctly get a packet of data if it is in the emission radius of the sender node. In a real environment, this assumption is not always true, due to many factors like signal attenuation and presence of obstacles. In what follows, we list the most known broadcasting methods according to their environment assumption.

\subsubsection{UDM-based broadcasting}

- MPR [2]: First proposed by Qayyum and al., this method treats broadcasting from the sink node to all nodes in a network and it is based on levels (i.e., the sink's neighbors are considered as level one nodes, the level one nodes' neighbors are considered as level two nodes). So, the broadcasting is performed by levels and the method choose each time the best nodes to relay. These nodes are a subset of a unique level nodes. The selection of relaying nodes is performed according to many factors. The aim of the MPR method is to minimize the redundant packets and the cost of communication, but it suffers from some problems because it is conceived in an ideal environment which means that a packet of data is correctly sent from a node $\mathrm{A}$ to a node $\mathrm{B}$ if the distance between A and B doesn't exceed the emission radius of A. Since its apparition, this method has been widely studied. In [5], the authors propose Getaway MPR, which is an improvement of MPR. In [18], MPR is used to conceive a routing protocol.

- DS-MPR $[11,12]$ : This method is an improvement of MPR, in which the broadcasting process is based on the connected dominating set which contains the nodes that have the greatest weight in the network. This weight is computed for every node in the network as a 
function of node degree and node remaining energy. Therefore, using DS-MPR in broadcasting can increase the lifetime of the network. This was improved in [13] by defending an extension of MPR [22] to compute the dominating set. In [15], the authors propose an algorithm to minimize m-connected k-tuple dominating sets which help to realize a fault tolerant broadcasting.

- In $[20,21,23]$ the authors propose broadcasting algorithms in structured WSNs (i.e., WSNs which are organized in clusters). In such a network, clustering has been used to induce a hierarchical structure over a flat WSN which minimizes communication overhead. However, all these method stipulate the environment as ideal.

\subsubsection{LNM-based broadcasting}

- MPR with LNM: In [3, 17], there are propositions of MPR in a realistic environment. The given methods improve the reachability of the original heuristics. Thus, it provide a logic and realistic performance. In the proposed heuristics, the MPR algorithm is modified in order to maximize its performance.

- GHA: Greedy Heuristic Algorithm : In [4], the authors provide a broadcasting method using a realistic physical layer by adopting a probability of reception between nodes. The use of LNM induces a broadcasting method with acceptable reachability.

- DS-MPR with LNM: In [1], there is a simple proposition of DS-MPR in a realistic environment, aiming to increase its reachability in such an environment.

\subsubsection{Summary}

The use of LNM provide a realistic behavior to the methods conceived in UDM. According to the best of our knowledge, there is no proposition of DS-MPR with a realistic physical layer except in [1]. Therefore, we try to provide a new model to compute node weight in this paper. This model takes into account communication factors in a realistic environment.

\subsection{Notations and problem definition}

WSNs or ad-hoc network can be considered as a graph $G(V, E)$, where the nodes constitute the set of vertex $V$ and $E$ the set of edges. An edge 
Table 1: Notation and symbols

\begin{tabular}{|c|l|}
\hline Symbol & Signification \\
\hline$G(V, E)$ & Graph connectivity where $V$ is the set of vertex \\
& and $E$ is the set of edges. \\
$u$ & Initial sender node \\
$N 1(u)$ & The set of neighbors of a node $u$ (order 1 neighbors) \\
$N 2(u)$ & The set of neighbors of $u$ 's neighbors (order 2 neighbors) \\
$N 3(u)$ & order 3 neighbors of $u$ \\
$v$ & Element of $N 1(u)$ \\
$w$ & Element of $N 2(u)$ or $N 1(v)$ \\
$x$ & Element of $N 3(u)$ or $N 2(v)$ or $N 1(w)$ \\
$S(u)$ & Subset of $N 1(u)$ chosen to relay \\
$S 1(u)$ & Subset of $N 2(u)$ not covered yet by nodes in $S(u)$ \\
\hline
\end{tabular}

exists between the two nodes $\mathrm{A}$ and $\mathrm{B}$ in this graph if a packet sent from $\mathrm{A}$ can be received at $\mathrm{B}$ or from $\mathrm{B}$ to $\mathrm{A}$. In table 1 , we introduce the symbols used in this paper and their definitions.

In figure 1, we suppose the broadcast of a message from $u$ to all other nodes in the networks taking into account:

- The minimization of redundant packets

- The minimization of energy consumption

Resolving this problem by using the relay-based broadcasting method means choosing an optimal subset of $N 1(u)$ taking into consideration the previously mentioned criteria. This subset will resend the packet received from $u$ (not all nodes in $N 1(u)$ resend). Finally, the packet should reach nodes in $N 2(u)$ according to the optimality of the subset chosen. If the network has many levels the same strategy of choosing a subset to relay is executed in every step. In the next section, we make an overview of a solution for this problem [11] (which is DS-MPR), and we show its limitation in a realistic environment.

\section{DS-MPR: An overview}

\section{1. $D S-M P R$ heuristic}

As describe above, the DS-MPR broadcasting process is based on a connected dominating set which contains the nodes having the greatest weight 


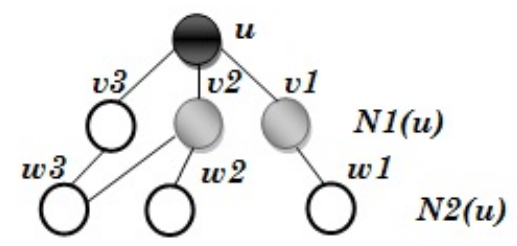

Figure 1: Example of a network: the initial sender node is black and the relay nodes are gray

in the network. This weight is a function of node degree and node remaining energy $(1,2)$.

$$
W(u)=\alpha \times W_{\text {Degree }}(u)+\beta \times W_{\text {Energy }}(u)
$$

With:

$$
\alpha+\beta=1
$$

To choose the optimal subset of $N 1(u)$, the algorithm below is applied (we have made a slight moderation to the original algorithm).

\begin{tabular}{l} 
Algorithm 1: $\mathbf{D S}$ - $M P R$ heuristic \\
\hline 1. Start with an empty set $S(u)$ and $S 1(u)=N 2(u)$. \\
2. First select nodes in $S 1(u)$ which have a single parent in $N 1(u)$ \\
then remove them from $S 1(u)$ and add their parents to $S(u)$. \\
3. While there still exist some nodes in $S 1(u)$ : \\
(a) Compute the weight of all nodes in $N 1(u) \backslash S(u)$ \\
(b) Add node $v$ to $S(u)$ for which this weight is maximum. If there \\
is more than one node with the same maximum weight choose the \\
one which has the maximum remaining energy. Then remove $N 1(v)$ \\
from $S 1(u)$.
\end{tabular}

This heuristic method is applied in UDM, and in the next subsection, we evaluate it into LNM, and we show the difference of accessibility of DS-MPR in these two models.

\subsection{Lognormal model}

With a realistic physical layer a reception cannot be performed as in a unit disk model due to many factors like signal attenuation, obstacle presence, distance between the sender, and the receiver, or communication time slot 
Table 2: Number of nodes according to the transmission radius

\begin{tabular}{|c|c|c|c|c|c|}
\hline$R=21 m$ & $R=20 m$ & $R=19 m$ & $R=18 m$ & $R=17 m$ & $R=16 m$ \\
\hline \multicolumn{7}{|c|}{$\begin{array}{c}\text { Number of nodes in line }=\frac{250}{(2 \times R)}, \text { we suppose } \\
\text { that the distance between nodes is the same }\end{array}$} \\
\hline 5.95 & 6.25 & 6.85 & 6.94 & 7.35 & 7.81 \\
\hline \multicolumn{7}{|c|}{ Number of nodes in area $=\left(\frac{250}{2 \times R}\right)^{2}$} \\
\hline 35.43 & 39.06 & 43.28 & 48.22 & 54.06 & 61.03 \\
\hline \multicolumn{7}{|c|}{ Total nodes in the field } \\
\hline $6 \times 5=30$ & $6 \times 6=36$ & $6 \times 7=42$ & $7 \times 7=49$ & $7 \times 8=56$ & $8 \times 8=64$ \\
\hline
\end{tabular}

(day or night). Therefore, we find the lognormal model (3), which is defined below, as a probability function according to most of these factors $[3,4]$.

$$
P(x)=\left\{\begin{array}{ccc}
1-\frac{\left(\frac{x}{R_{c}}\right)^{2 \alpha}}{2} & \text { if } & 0 \prec x \preceq R_{c} \\
\frac{\left(\frac{2 R_{c}-x}{R_{c}}\right)^{2 \alpha}}{2} & \text { if } \quad R_{c} \prec x \preceq 2 R_{c} \\
0 & \text { otherwise }
\end{array}\right.
$$

Below, we describe the symbols used:

$\alpha$ : Signal attenuation factor.

$R_{c}$ : Sender node transmission radius.

$x$ : Distance between sender and receiver nodes

\section{3. $D S-M P R$ illustration in a realistic environment}

The test was performed using NS2 [14]. We worked in a field of $250 \mathrm{~m} \times$ $250 \mathrm{~m}$, with nodes that have $25 \mathrm{~m}$ as transmission radius. The average distance between nodes according to their density is given in figure 2. We chose an average between $21 \mathrm{~m}$ and $16 \mathrm{~m}$, which is real in our opinion. The average distance starts at $21 \mathrm{~m}$ for 30 nodes and becomes $16 \mathrm{~m}$ for 64 nodes in the field. In table 2, we try to compute the number of nodes in a field of $250 m \times 250 m$ according to a transmission radius average between $21 m$ and $16 m$. 


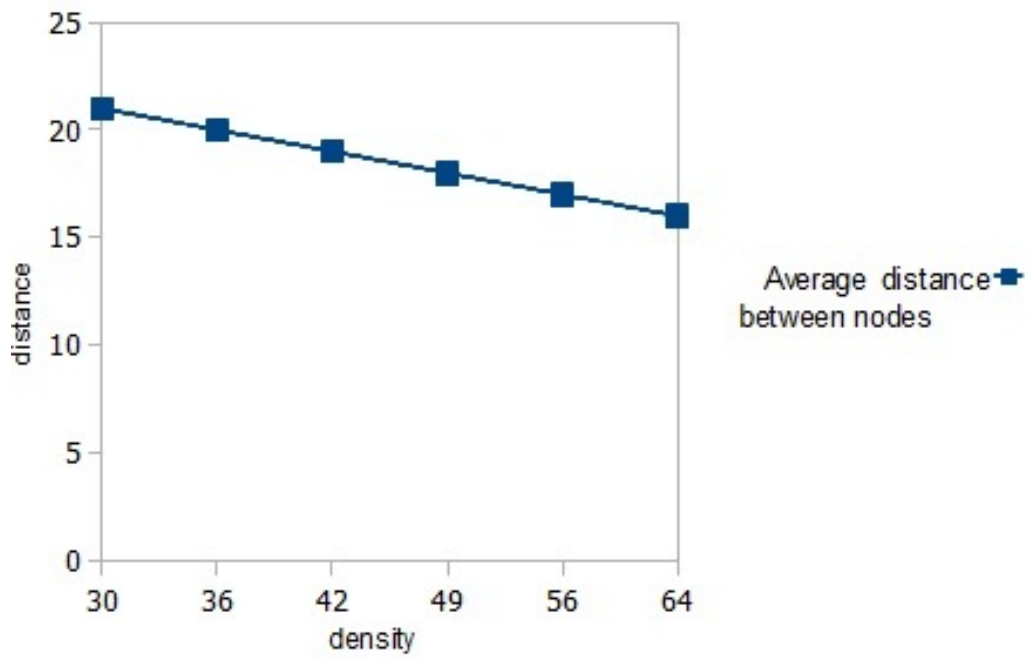

Figure 2: Average distance between nodes according to the density

Figure 2 describes the number of nodes according to the transmission radius chosen.

In figure 3, we make a comparison between probabilities of reception in UDM and in LNM for a transmission radius $R c=25$. Consequently, we can find a difference due to that in UDM the probability of reception is equal to 1 if the distance between the sender and the receiver is less than 25 and equal to 0 if otherwise. However, in LNM, the probability of reception is computed according to $(3)$.

In figure 4, we make a comparison between the accessibility of DS-MPR in the two models according to figure 2 and figure 3 . We can see that in an ideal environment DS-MPR looks as ideal with an accessibility of $100 \%$ due to the fact that a packet of data is certainly received when the distance between the sender and a receiver doesn't exceed the transmission radius. However, in a realistic environment, this accessibility started at less than $58 \%$ and can reach $80 \%$ in the best case. With more than 64 nodes, we can see that the curve of accessibility becomes almost linear. So, this method looks invalid in LNM because its reachabilty cannot exceed $80 \%$. In the next section, we propose a modification of DS-MPR to be applicable with a realistic physical layer. 


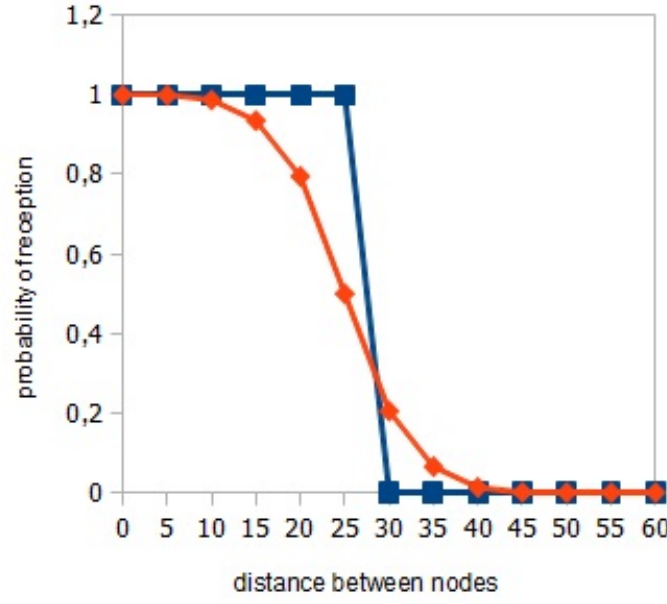

$\mathrm{Rc}=25:$ lognormal +

$\mathrm{Rc}=25$ : unit disk $\rightarrow$

Figure 3: Comparison of probability of reception between unit disk model and lognormal model for a transmission radius $=25 \mathrm{~m}$

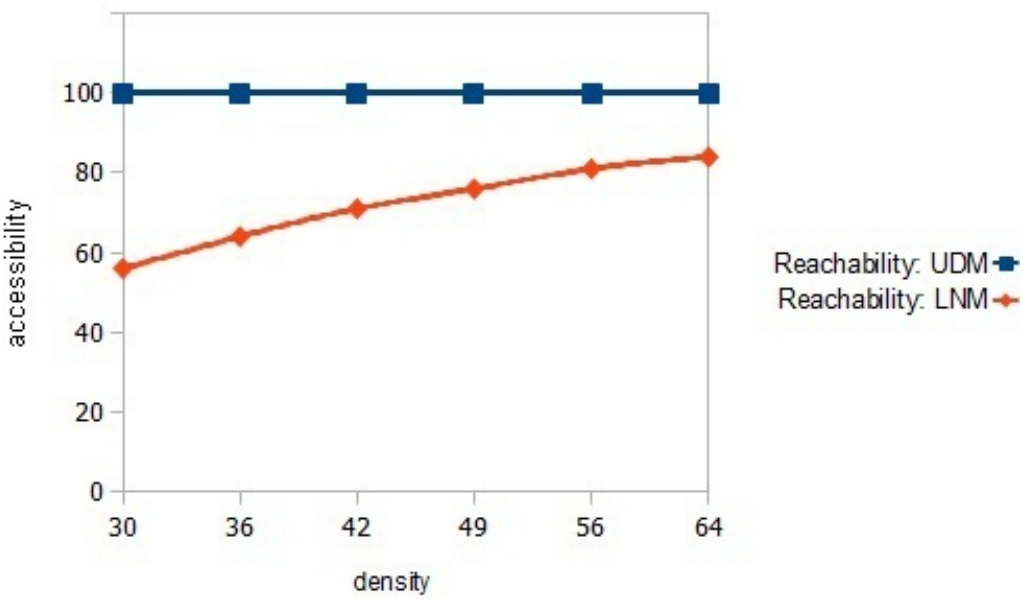

Figure 4: Comparison of DS-MPR accessibility between unit disk model and lognormal model 


\section{DS-MPR in a realistic environment}

Connected Dominating Sets with multiPoint relay is a method of broadcasting where a weight for each node in the network is computed. This weight is a function of node remaining energy and node degree. Then, a set of nodes to relay packets is chosen. This set consists of nodes that have the greatest weight and should provide good reachability. Furthermore, this method is conceived in an ideal environment and, in what follow, we try to define it with a realistic physical layer.

\subsection{RDS-MPR heuristic}

In this heuristic, we propose a new weight function, called $R W$ (Realistic Weight $(4,5))$, which is changed from DS-MPR (2) to RDS-MPR, taking into account the reception probability according to the lognormal model (3):

$$
R W(u)=\alpha \times R W_{\text {Degree }}(u)+\beta \times R W_{\text {Energy }}(u)+\gamma \times R W_{\text {Probability }}(u)
$$

with

$$
\alpha+\beta+\gamma=1
$$

(i) The realistic weight degree of a node $v(6)$ is the number of nodes reachable by $v$ and not reachable yet by another node in $S(u)$ divided by the maximum degree weight of the nodes in $N 1(u) \backslash S(u)$.

$$
R W_{\text {Degree }}(v)=\frac{|N 1(v) \bigcap S 1(u)|}{\operatorname{Max}\left\{\left|N 1\left(v_{i} \bigcap S 1(u)\right)\right| ; v_{i} \in N 1(u) \backslash S(u)\right\}}
$$

(ii) The realistic weight energy of a node $v(7)$ is the remaining energy in $v$ divided by the maximum weight energy of the nodes in $N 1(u) \backslash S(u)$.

$$
R W_{\text {Energy }}(v)=\frac{E(v)}{\operatorname{Max}\left\{E\left(v_{i}\right) ; v_{i} \in N 1(u) \backslash S(u)\right\}}
$$

(iii) The realistic weight probability of a node $v(8)$ is a local weight probability $q(v)(9)$ divided by the maximum local weight probability of the nodes in $N 1(u) \backslash S(u)$.

$$
R W_{\text {Probability }}(v)=\frac{q(v)}{\operatorname{Max}\left\{q\left(v_{i}\right) ; v_{i} \in N 1(u) \backslash S(u)\right\}}
$$


(iv) This local probability $q(v)(9)$ computes the $v$ node weight according to the probability of reception from $u$ to $v$ and from $v$ to its neighbors.

$$
q(v)=\left\{\begin{array}{cc}
p(u, v) \times \frac{\sum_{i=1}^{|N 1(v) \cap S 1(u)|} p\left(v, w_{i}\right)}{|N 1(v) \bigcap S 1(u)|} & i f|N 1(v) \bigcap S 1(u)| \neq 0 \\
0 & \text { otherwise }
\end{array}\right.
$$

After giving the definition of every weight in the new weight function, we have written our heuristic bellow. We recall that:

$p t$ : is a temporary probability in the heuristic and with it, a reception can be performed well.

$p 0$ : is the minimum probability of a good reception. With less than $p 0$, we consider the data received is wrong or the reception was not performed.

\section{Algorithm 2: RDS-MPR heuristic}

1. Start with an empty set $S(u)$ and $S 1(u)=N 2(u)$.

2. First select nodes in $S 1(u)$ which have a single parent in $N 1(u)$ then remove them from $S 1(u)$ and add their parents to $S(u)$.

3. While there still exist some nodes in $S 1(u)$ :

(a) Compute the weight of all nodes in $N 1(u) \backslash S(u)$

(b) Add node $v$ to $S(u)$ for which this weight is maximum and $p(u, v)>p t$. If there is more than one node with the same maximum weight choose the one which has the maximum remaining energy. Then remove $N 1(v)$ from $S 1(u)$.

(c) Compute reachability $R e$.

(d) If $R e<90 \%$ and $p t>p 0$ then slightly reduce $p t$ and slightly increase $\gamma$

Else break.

\subsection{RDS-MPR illustration}

To evaluate our heuristic, we test it with the same density used to test DS-MPR. In figure 5, we can see that RDS-MPR provides a good accessibility with $\gamma=1 / 3$ and $p 0=0.6$ This accessibility started near $80 \%$ with a density 


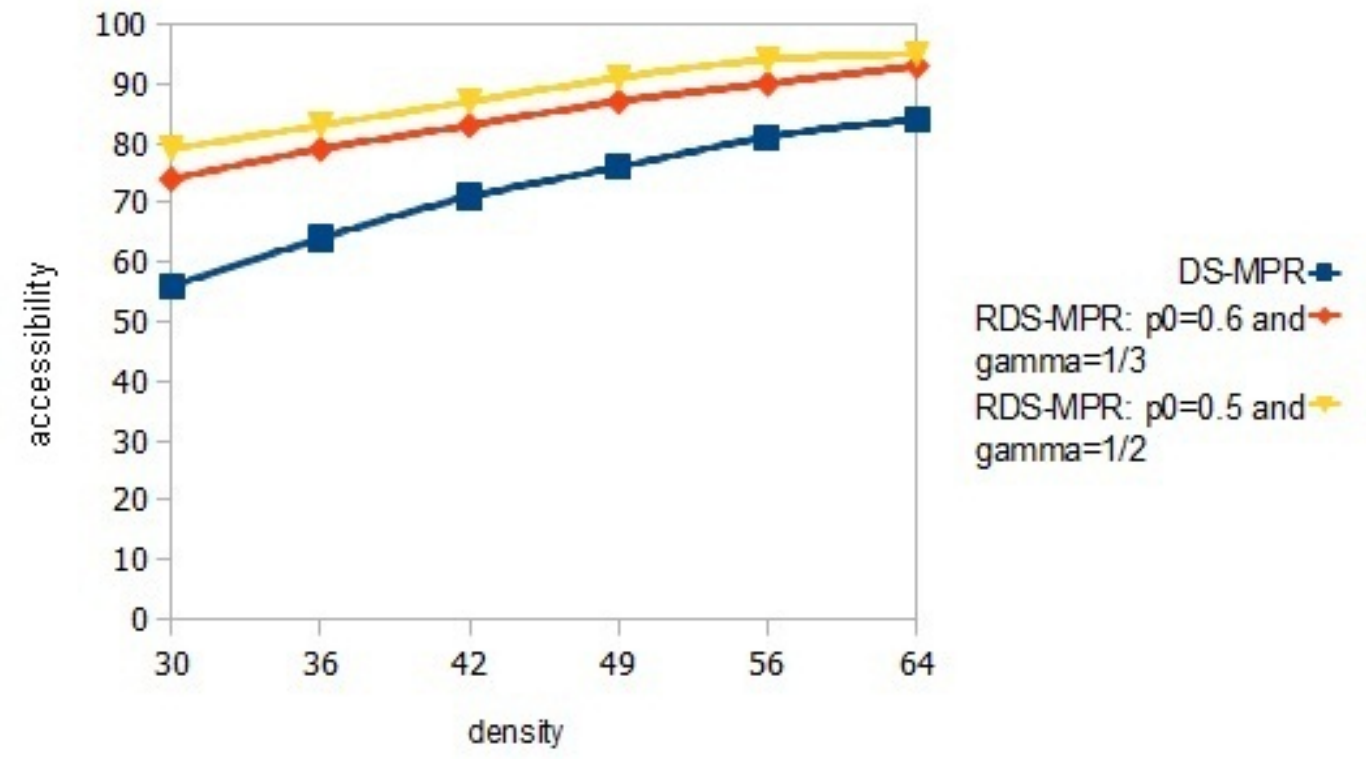

Figure 5: Comparison of the accessibility in lognormal model between DS-MPR and RDSMPR for $\gamma=1 / 3, p 0=0.6$ and for $\gamma=1 / 2, p 0=0.5$

of 30 nodes and becomes more than $90 \%$ for a density with 64 nodes. With $\gamma=1 / 2$ and $p 0=0.5$, our heuristic provides a good accessibility that become more than $94 \%$ with 64 nodes as density.

\section{Extended DS-MPR in a realistic environment}

\section{1. eRDS-MPR heuristic}

In this subsection, we define another broadcasting method which is an extension of RDS-MPR. We try to change the node weight by making it deep. What we mean by deep is changing the node degree weight, the node energy weight and the node probability weight by taking into account $N 2(u)$ and $N 3(u)$ (see figure 6). First, the extended nodes weight $E W(10,11)$ is defined.

$$
E W(u)=\alpha \times E W_{\text {Degree }}(u)+\beta \times E W_{\text {Energy }}(u)+\gamma \times E W_{\text {Probability }}(u)
$$




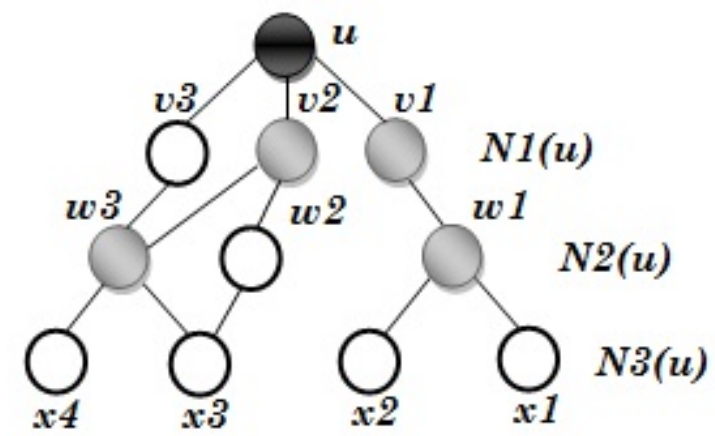

Figure 6: Example of WSNs: the initial sender node is black and nodes chosen to relay are gray; we also define $N 1(u), N 2(u)$ and $N 3(u)$.

With:

$$
\alpha+\beta+\gamma=1
$$

Every weight in the function above is defined below:

(i) The extended weight of a node degree $v(12)$ is the weight of $v$ given in (13) divided by the maximum of the same weight (13) of nodes in $N 1(u) \backslash S(u)$.

$$
E W_{\text {Degree }}(v)=\frac{E W_{\text {Degree }}^{\prime}(v)}{\operatorname{Max}\left\{E W_{\text {Degree }}^{\prime}\left(v_{i}\right) ; v_{i} \in N 1(u) \backslash S(u)\right\}}
$$

With:

$$
E W_{\text {Degree }}^{\prime}(v)=\left\{\begin{array}{cc}
|N 1(v) \bigcap S 1(u)| \times \frac{\sum_{i=1}^{|N 1(v) \bigcap S 1(u)|}\left|N 1\left(w_{i}\right)\right|}{|N 1(v) \bigcap S 1(u)|} & \text { otherwise } \\
0 & \text { if }|N 1(v) \bigcap S 1(u)| \neq 0
\end{array}\right.
$$

The previous weight computes the number of nodes that can be reached by $v$ and not reached yet by node of $N 1(u)$ multiplied by the average of nodes that can be reachable by $w_{i}\left(w_{i}=N 1(v)\right)$. Consequently, this weight helps us to choose nodes having the best reachability for the next level ( $w_{i}$ nodes), in addition to the level after ( $x_{i}$ nodes). 
(ii) The extended weight of a node energy $v(14)$ is the weight of $v$ given in (15) divided by the maximum of the same weight (15) of nodes in $N 1(u) \backslash S(u)$.

$$
E W_{\text {Energy }}(v)=\frac{E W_{\text {Energy }}^{\prime}(v)}{\operatorname{Max}\left\{E W_{\text {Energy }}^{\prime}\left(v_{i}\right) ; v_{i} \in N 1(u) \backslash S(u)\right\}}
$$

with:

$$
E W_{\text {Energy }}^{\prime}(v)=\left\{\begin{array}{cc}
E(v) \times \frac{\sum_{i=1}^{|N 1(v) \bigcap S 1(u)|} E\left(w_{i}\right)}{|N 1(v) \bigcap S 1(u)|} & i f|N 1(v) \bigcap S 1(u)| \neq 0 \\
0 & \text { otherwise }
\end{array}\right.
$$

This weight computes the remaining energy in $v$ multiplied by the average of remaining energy of $w_{i}$. We choose only nodes which is not reachable yet (i.e., $w_{i}$ in $N 1(v) \bigcap S 1(u)$ ). Consequently, this weight helps us to choose nodes having the maximum remaining energy and which can be reached. This weight allows also the selection, in the next level, of nodes having the maximum average remaining energy. As well as increasing the reachability, the proposed weight can increase the lifetime of the network because the node with the maximum remaining energy is used first.

(iii) The extended weight of a node probability $v(16)$ is the weight of $v$ given in (17) divided by the maximum of the same weight (17) of nodes in $N 1(u) \backslash S(u)$.

$$
E W_{\text {Probability }}(v)=\frac{E W_{\text {Probability }}^{\prime}(v)}{\operatorname{Max}\left\{E W_{\text {Probability }}^{\prime}\left(v_{i}\right) ; v_{i} \in N 1(u) \backslash S(u)\right\}}
$$


With:

$$
E W_{\text {Probability }}^{\prime}(v)=\left\{\begin{array}{c}
p(u, v) \times \frac{\sum_{i=1}^{|N 1(v) \cap S 1(u)|} p\left(v, w_{i}\right) \times \frac{\sum_{j=1}^{\left|N 1\left(w_{i}\right)\right|} p\left(w_{i}, x_{j}\right)}{\left|N 1\left(w_{i}\right)\right|}}{|N 1(v) \bigcap S 1(u)|} \\
i f|N 1(v) \bigcap S 1(u)| \times\left|N 1\left(w_{i}\right)\right| \neq 0
\end{array}\right.
$$

0

otherwise

We define this weight in order to choose the route with the best probability. This route starts from $u$ to $v$ afterward, from $v$ to $w$ and finally, from $w$ to $x$. Consequently, this weight computes $p(u, v)$ multiplied by the average of probability from $v$ to $w_{i}$ multiplied by the average of probability from $w_{i}$ to $x_{j}$.

By analogy to the RDS-MPR heuristic, below, we introduce our eRDSMPR heuristic and we recall that:

$p t$ : is a temporary probability in the heuristic. With it, a reception can be performed well.

$p 0$ : is the minimum probability of a good reception. With less than $p 0$, we consider that the data received is wrong or the reception was not performed. 


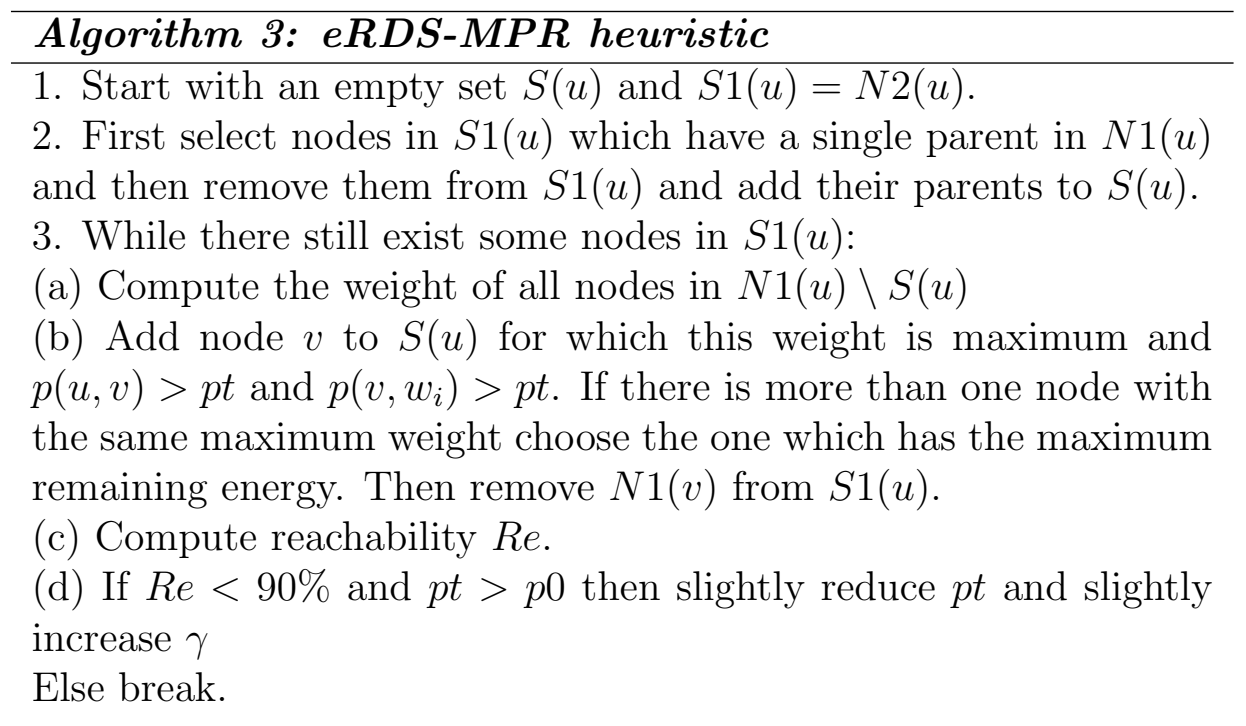

\section{2. eRDS-MPR illustration}

To evaluate our heuristic, we test it with the same density used to test DS-MPR and RDS-MPR. In figure 7, we can see that RDS-MPR provides a good accessibility with $\gamma=1 / 3$ and $p 0=0.6$. This accessibility starts near $80 \%$ with a density of 30 nodes and becomes more than $90 \%$ for a density with 64 nodes. With $\gamma=1 / 3$ and $p 0=0.5$, RDS-MPR provides a high accessibility that becomes more than $94 \%$ with 64 nodes as density. However, the eRDS-MPR heuristic tested with $\gamma=1 / 3$ and $p 0=0.6$ gives a reachability starts at $87 \%$ with 30 nodes and can exceed more than $97 \%$ with a density of 60 nodes. We can conclude here that our method is valid and makes an important improvement in the term of reachability if we accept that a broadcasting method is considered valid when it provides more than $90 \%$ reachability.

\section{Performance evaluation}

In this section a performance comparison between DS-MPR, RDS-MPR and eRDS-MPR is given. This comparison treats the consumed energy, and the use of nodes resources in each method. 


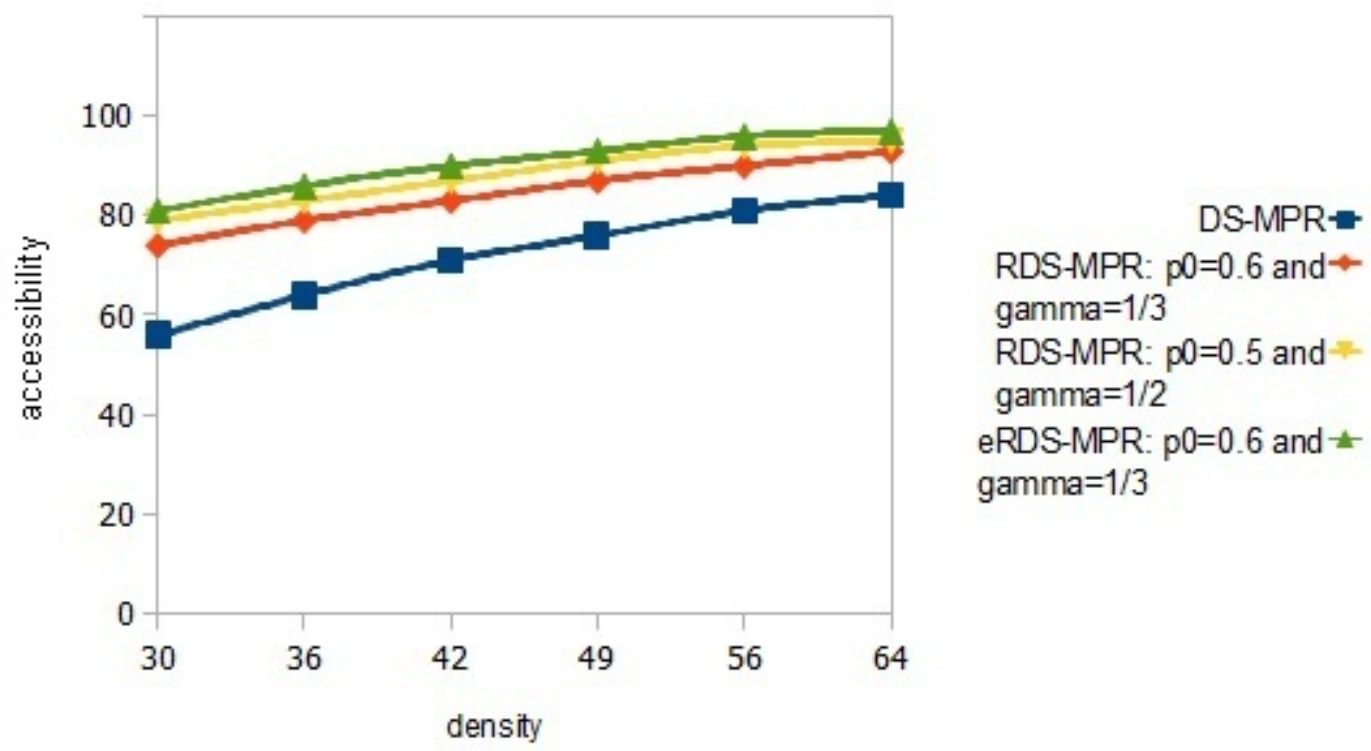

Figure 7: Comparison of the accessibility in lognormal model between DS-MPR and RDSMPR for $\gamma=1 / 3, p 0=0.6$ and for $\gamma=1 / 2, p 0=0.5$ and eRDS-MPR for $\gamma=1 / 3$, $p 0=0.6$ 


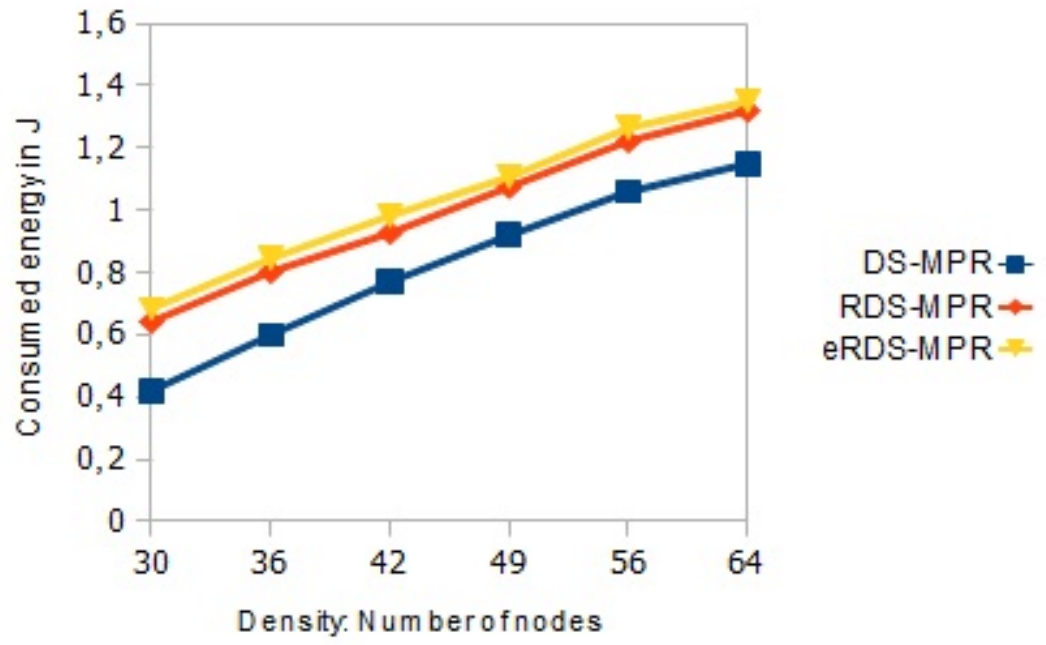

Figure 8: Comparison of consumed energy to accomplish broadcasting between DS-MPR $(\alpha=1 / 2$ and $\beta=1 / 2)$, RDS-MPR and eRDS-MPR $(\alpha=1 / 3, \beta=1 / 3, \gamma=1 / 3$ and $p 0=0.6)$

\subsection{Comparison of the consumed energy}

In figure 8, a comparison of consumed energy to accomplish the broadcasting task is provided. The simulation is performed on networks with different densities. The packet size used to test is $200 b$. It is clear that increasing reachability causes more energy consumption. In fact, we have to exchange more messages to appreciate the state of nodes and to reach more sensors. However, In DS-MPR there is a lot of lost energy used to send packets to unreachable nodes. For example, if we consider a network with 64 nodes:

- According to figure 8, RDS-MPR and eRDS-MPR increase the energy consumption of DS-MPR respectively with $\frac{1.32-1.15}{1.15}=14.7 \%$ and $\frac{1.35-1.15}{1.15}=17.3 \%$.

- According to figure 6, RDS-MPR and eRDS-MPR increase the reachability of DS-MPR respectively with $\frac{94-80}{80}=17.5 \%$ and $\frac{97-80}{80}=21.2 \%$.

Thus, we can conclude that the augmentation of reachability is performed with less amount of energy. 


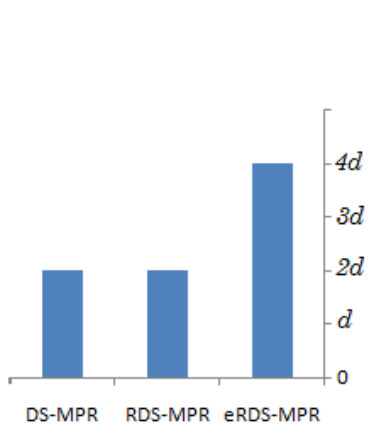

(a)

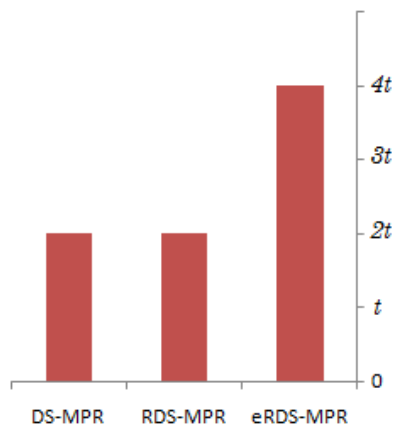

(b)

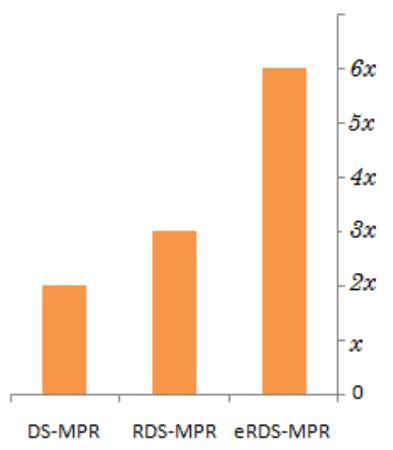

(c)

Figure 9: Performance comparison, (a): Exchanged messages, (b): Communication time and (c): Memory size

\subsection{Performance comparison}

In this subsection, we compare the performance of three approaches in term of exchanged message, communication time and memory used.

We introduce the following notations (figure 9):

- $d$ : The average degree of nodes in the graph

- $t$ : The average time which is necessary to perform a communication between two nodes

- $x$ : the average size to save data concerning a weight in node memory

We assume in our case that all nodes have the same proprieties in term of exchanged messages, communication time and data size.

Figure 9(a) describes the average number of necessary messages to be exchanged for collecting data in order to compute weights in each methods, whereas figure 9 (b) describes the average of necessary wait time for collecting data used in the weight formula before computing weights in each methods. Finally, figure 9 (c) describes the average memory size necessary for saving data for each weight. Figure 9 provides a performance comparison in term of messages number to be exchanged, waiting time for necessary data and memory size in which necessary data for computing weights will be saved. The measurements are based on level average in the network. We notice that values in the eRDS-MPR method are doubled compared to RDS-MPR due to the levels number, 
yet, exchanged messages and waiting time values of RDS-MPR are the same compared to DS-MPR except for the memory size needed, because RDS-MPR have one extra weight. Finally, although eRDS-MPR provides an improvement of RDS-MPR in reachability, this comparison indicates that RDS-MPR is better than eRDS-MPR in term of optimizing the use of node resources in the network.

\section{Conclusion}

Many algorithms of broadcasting in WSN and Ad-Hoc networks were focused on solving problems caused by pure flooding (i.e., redundant data, packet collision and important energy consumption). Most of these solutions model communications are in ideal environments, adopting the unit disk model. In this hypothesis, algorithms are not valid. We consider that a method of broadcasting with reachability less than $90 \%$ is not enough. Therefore, researchers propose new methods taking care of nature's factor. In this paper, we adapt the DS-MPR broadcast algorithm, to be applied in a more realistic environment, taking into account packet loss and unreliable communication links. Our proposal is RDS-MPR, which improves DS-MPR and provides accessibility at more than 94\%. We propose also eRDS-MPR, which can give us a reachability that can exceed $97 \%$ in the best case and no less than $87 \%$ in the worst case. Finally, we compare the performance of the three approaches in term of consumed energy, exchanged message, communication time and memory used.

\section{References}

[1] Tarek Moulahi, Hervé Guyennet, Mohamed Lehsaini, and Salem Nasri. An Energy Aware MPR-based Broadcasting Algorithms for Wireless Sensor Networks: International Conference on Selected Topics in Mobile and Wireless Networking (iCOST); 2011. p.13-18.

[2] Amir Qayyum, Laurent Viennot, and Anis Laouiti. Multipoint Relaying for Flooding Broadcast Messages in Mobile Wireless Networks: Proceedings of the 35th Hawaii International Conference on System Sciences; 2002. p.3866-3875.

[3] Mohamed Lehsaini, Hervé Guyennet, and Mohamed Feham. MPRbased broadcasting in ad hoc and wireless sensors networks with a 
realistic environment: IJCSNS International Journal of Computer Science and Network Security; 2007. p.82-89.

[4] Gary K.W.Wong, Hai Liu, Xiaowen Chu, Yiu-Wing Leung, and Chun Xie. Efficient broadcasting in multi-hop wireless networks with a realistic physical layer: Ad Hoc Networks; 2010.

[5] Ou Liang, Y.Ahmet Sekercioglu, and Nallasamy Mani. Gateway multipoint relays an-MPR-based broadcast algorithm for ad hoc networks: IEEE Singapore International Conference on Communication systems; 2006. p. 1-6.

[6] Chih-Shun Hsu, Jang-Ping Sheu, and Yen-Jung Chang. Efficient Broadcasting Protocols for Regular Wireless Sensor Networks: International Conference on Parallel Processing; 2003. p. 213-220.

[7] Chien-Chun Ni, Tien-Ruey Hsiang, and J.D. Tygar. A PowerPreserving Broadcast Protocol for WSNs With DoS Resistance: Proceedings of International IEEE Conference on Computer Communications and Networks; 2008. p. 1-6.

[8] Brad Williams, and Tracy Camp. Comparison of Broadcasting Techniques for Mobile Ad Hoc Networks: Proceedings of the ACM International Symposium on Mobile Ad Hoc Networking and Computing; 2002. p. 194-205.

[9] L. Orecchia, A. Panconesi, C. Petrioli, and A. Vitaletti. Localized Techniques for Broadcasting in Wireless Sensor Networks: Proceedings of the 2004 joint workshop on Foundations of mobile computing; 2004. p. 41-51.

[10] Deng Yubo, Niu Jianwei and Li Lian. Modeling of Broadcasting based on Distance Scheme for WSN: International Joint Conference on INC, IMS and IDC; 2009. p. 1176-1179.

[11] C. Adjih, P. Jaquet, and L. Viennot. Computing Connected Dominated Sets with Multipoint Relays: International Journal Ad Hoc \& Sensor Wireless Networks; 2005. p. 27-39.

[12] Xiao Chen, and Jian Shen. Reducing Connected Dominating Set Size with Multipoint Relays in Ad Hoc Wireless Networks: Proceedings of the International Symposium on Parallel Architectures, Algorithms and Networks; 2004. p. 539-543.

[13] Jie Wu, Wei Lou, and Fei Dai. Extended Multipoint Relays to Determine Connected Dominating Sets in MANETs: IEEE transactions on computers; 2006. p. 334-347. 
[14] Imad Aad, Mohammad Hossein Manshaei, and JeanPierre Hubaux. NS-2 for the impatient: EPFL Lausanne Switzerland; 2009.

[15] Weiping Shang, Pengjun Wan, Frances Yao, and Xiaodong Hu. Algorithms for minimum m-connected $\mathrm{k}$-tuple dominating set problem: Theoretical Computer Science; 2007. p.241-247.

[16] Ananth V. Kini, Nikhil Singhal, and Steven Weber. Broadcast capacity of a WSN under communication and information coordination: Ad Hoc Networks; 2010. p. 439455.

[17] Francois Ingelrest, and David Simplot-Ryl. Maximizing the Probability of Delivery of Multipoint Relay Broadcast Protocol in Wireless Ad Hoc Networks with a Realistic Physical Layer: Mobile Ad hoc and Sensor Networks, Lecture Notes in Computer Science (Springer); 2006. p.143154.

[18] Anthony Busson, Nathalie Mitton, and Eric Fleury. An analysis of the Multi-Point Relays selection in OLSR: RR-5468,INRIA; 2005.

[19] Pere Montolio-Aranda, Joaquin Garcia-Alfaro, and David Megias. Improved flooding of broadcast messages using extended multipoint relaying: Journal of Network and Computer Applications; 2011. p. 542-555.

[20] E Sivaraman. Dynamic Cluster Broadcasting for Mobile Ad Hoc Networks: Proceedings of the International Conference on Communication and Computational Intelligence; 2010. p. 123-127.

[21] Long Cheng, Sajal K. Das, Mario Di Francesco, Canfeng Chen, and Jian Ma. Scalable and Energy-Efficient Broadcasting in Multi-hop Cluster-Based Wireless Sensor Networks: IEEE ICC 2011 proceedings; 2011. p. 1-5.

[22] Pere Montolio-Arandaa, Joaquin Garcia-Alfaroa, and David Megias. Improved flooding of broadcast messages using extended multipoint relaying: Journal of Network and Computer Applications; 2011. p. 542-555.

[23] Wei chen, A.K.M.Muzahidul Islam, Mohan Malkani, Amir Shirkhodaie, Koichi Wada, and Mohamed Zein-Sabatto. Novel Broadcast/Multicast Protocols for Dynamic Sensor Networks: IEEE International Parallel and Distributed Processing Symposium; 2007. p. 1-8.

[24] Wahabou Abdou, Adrien Henriet, Christelle Bloch, Dominique Dhoutaut, Damien Charlet, and Franois Spies. Using an Evolutionary Algorithm to Optimize the Broadcasting Methods in Mobile Ad hoc Networks: Journal of Network and Computer Applications; 2011. pp. 1794-1804. 
[25] Javad Akbari Torkestani, and Mohammad Reza Meybodi. A link stability-based multicast routing protocol for wireless mobile ad hoc networks: Journal of Network and Computer Applications; 2011. p. 1429-1440. 